\title{
Relationship between the endothelium biomarkers endocan and thrombomodulin and slow coronary flow.
}

\author{
Sadettin Selçuk Baysal ${ }^{1 *}$, Şahbender Koç², Bedri Caner Kaya ${ }^{1}$, Ahmet Güneş ${ }^{1}$, Naime Meriç Konar³, \\ İbrahim Halil Altiparmak ${ }^{4}$ \\ ${ }^{1}$ Cardiology Department, Şanlıurfa Mehmet Akif İnan Training and Research Hospital, Şanlıurfa, Turkey \\ ${ }^{2}$ Cardiology Department, Keçiören Training and Research Hospital, Ankara, Turkey \\ ${ }^{3}$ Department of Biostatistics, Hacettepe University, Ankara, Turkey \\ ${ }^{4}$ Department of Cardiology, Harran University, Şanlıurfa, Turkey
}

\begin{abstract}
Background: Growing evidence suggests that endothelial dysfunction plays a key role in the pathophysiology of Slow Coronary Flow (SCF). This study investigated the association between endothelial biomarkers endocan and soluble Thrombomodulin (sTM) and SCF.

Methods: The study population consisted of 89 subjects ( 54 patients with SCF and 35 controls). Coronary flow rate was assessed using the Thrombolysis in Myocardial Infarction (TIMI) Frame Count (TFC) method. The serum endocan and sTM levels of all subjects were analysed.

Results: Significantly higher endocan levels $(1.14 \pm 0.22 \mathrm{vs} .0 .96 \pm 0.32 \mathrm{ng} / \mathrm{ml} ; \mathrm{p}=0.009)$ and sTM levels $(657.06 \pm 198.18$ vs. $592.76 \pm \mathbf{1 2 8 . 4 5} \mathrm{pg} / \mathrm{ml} ; \mathrm{p}=\mathbf{0 . 1 1 9})$ were observed in the SCF group relative to the control group. A positive correlation was detected between endocan and TFC $(r=0.563 ; p=0.016)$. Multivariate logistic regression analysis revealed endocan as a predictor of SCF.

Conclusions: The endocan level in SCF patients was significantly higher than in controls and the STM level was also increased, although the difference was not significant. These results suggest that endocan may serve as a biomarker to predict SCF.
\end{abstract}

Keywords: Slow coronary flow, Endocan, Thrombomodulin, Endothelial dysfunction.

Accepted on January 19, 2018

\section{Introduction}

The Slow Coronary Flow (SCF) phenomenon is an angiographic finding described as retarded flow of contrast agent to the end branch of a coronary artery without significant coronary stenosis [1]. Although it is well-known among interventional cardiologists that coronary angiography is performed in approximately $1 \%$ of patients, the mechanisms underlying this entity have not yet been elucidated [2]. Microvascular dysfunction, endothelial dysfunction, diffuse atherosclerosis, and platelet and vasomotor dysfunction have been proposed in the etiology of SCF $[3,4]$.

Endothelial cell-specific molecule-1, or endocan, is a soluble proteoglycan secreted by human vascular endothelial cells and can be found in the circulation [5]. Previous studies have suggested that endocan plays a key role in the pathophysiology of endothelial dysfunction $[5,6]$.

Thrombomodulin (TM) is an integral membrane type-1 glycoprotein that is widely distributed on the lumenal surface of vascular endothelial cells and has been implicated in the endothelial regulation of fibrinolysis and coagulation. Soluble
Thrombomodulin (sTM) can be detected in serum after proteolysis of TM on the endothelium [7]. Previous studies have suggested that the sTM concentration reflects the degree of endothelial damage $[7,8]$.

Because endothelial dysfunction plays an important role in the pathophysiology of SCF, we aimed to determine whether sTM and endocan, two endothelial dysfunction biomarkers, are associated with SCF. Therefore, we investigated the relationship between these two biomarkers and SCF. Few studies have investigated the relationship between endocan and SCF; to the best of our knowledge, this was the first study to measure the concentration of STM in the circulation of SCF patients.

\section{Subjects and Methods}

This was a cross-sectional study, in which 6,517 patients underwent coronary angiography between February 2015 and January 2016. During this period, a total of 89 patients with an initial diagnosis of stable angina pectoris, and who had angiographically confirmed normal coronary arteries without 
any atherosclerotic lesions and SCF, were enrolled in the study. These subjects were divided into two groups based on flow rate: 54 patients with isolated $\mathrm{CSF}$ and 35 subjects with Normal Coronary Flow (NCF) were included in the final analysis.

Exclusion criteria were as follows: acute coronary syndromes, coronary ectasia (dilatation of the coronary artery diameter 1.5 times or more compared to the nearest normal segment) or anomaly, coronary vasospasm, left ventricular dysfunction (ejection fraction $<50 \%$ ), significant valvular disease, malignancy, acute or chronic inflammatory conditions, chronic obstructive lung disease, and liver, kidney, thyroid, or hematologic disorders. Demographic, clinical, and laboratory data of the subjects were obtained during their visit to the cardiology clinic before angiography. The study protocol was approved by the local ethics committee and all patients provided informed consent.

Coronary angiography was performed using the standard Judkins technique and $6 \mathrm{~F}$ catheters inserted through the femoral and radial arteries with a Siemens Axiom Sensis XP device (Siemens, Munich, Germany) at a rate of 30 frames per second. Iopromide (Ultravist 370; Schering AG, Berlin, Germany) was used as the contrast agent. Two cardiologists blinded to the study reviewed the angiograms and evaluated the coronary blood flow rates using the Thrombolysis in Myocardial Infarction (TIMI) frame count method (TFC) [9]. The intra- and inter-observer coefficients of variation were 5.2 and 7.8, respectively. The beginning frame was the first frame in which the contrast agent entered the artery completely. The last frame was defined as that when the contrast agent reached the distal endpoint of each vessel. The following distal endpoints of each coronary artery were reviewed: the distal bifurcation in the Left Anterior Descending artery (LAD), the distal bifurcation of the segment with the longest total distance in the Left Circumflex artery (LCX), and the first branch of the posterolateral artery in the Right Coronary Artery (RCA). A normal TFC is considered $36.2 \pm 2.6$ frames for LAD, $22.2 \pm$ 4.1 frames for LCX, and $20.4 \pm 3$ frames for RCA. The LAD frame count was divided by 1.7 to obtain a corrected TFC (cTFC) as the longer length of the LAD compared to other coronary arteries. The standard cTFC for the LAD coronary artery is $21.1 \pm 1.5$ frames. All subjects with a TFC greater than 2 standard deviations above the previously published range for the particular vessel were considered to have SCF [9]. In our study, SCF in any of the three coronary arteries was sufficient to include patients in the SCF group.

Venous blood samples were obtained from all subjects following a fasting period of $12 \mathrm{~h}$. All biochemical and hematologic values were taken on the day of sample collection. Hematologic measurements were determined using the Coulter LH 780 Hematology Analyzer (Beckman Coulter Inc., Co. Clare, Ireland). All biochemical parameters were determined using the Abbott Diagnostics C8000i auto-analyzer (Abbott, Wiesbaden, Germany). Blood samples were collected into plain tubes and serum was separated after centrifugation at $1,500 \mathrm{~g}$ for $10 \mathrm{~min}$ and stored at $-80^{\circ} \mathrm{C}$ until the levels of endocan and STM were analysed. Both serum endocan and sTM levels were measured using a sandwich enzyme-linked immunosorbent assay (ELISA) kit with high sensitivity and specificity for the detection of human endocan (Cusabio Bioscience Inc., Wuhan, China). The minimum detectable concentration of endocan and sTM was $0.039 \mathrm{ng} / \mathrm{ml}$ and 7.8 $\mathrm{pg} / \mathrm{ml}$, respectively. The intra- and inter-assay coefficients of variation were less than $8 \%$ and $10 \%$, respectively, for both biomarkers.

\section{Statistical analysis}

SPSS for Windows software (ver. 22.0; SPSS Inc., Chicago, IL, USA) was used for all statistical analyses. The ShapiroWilks test was used to evaluate whether the distributions of continuous variables were normal. The independent samples ttest was used to compare normally distributed continuous variables and, for non-normally distributed continuous variables, the Mann-Whitney U Test was used. Descriptive statistics, and mean and standard deviation values were used for the normally distributed variables. Median and minimummaximum values were used for non-normally distributed variables. Box-plot graphics are shown for biomarkers with a statistically significant difference between the SCF and NCF (control) groups. The correlations between the biomarkers and mean variables are shown. Spearman's and Pearson's correlation coefficients were used for non-normally distributed and normally distributed variables, respectively. Univariate and multivariate logistic regression analyses were performed to identify the major risk factors of SCF. Before multivariate logistic regression analysis, univariate analyses, such as the independent samples t-test, the Mann-Whitney U Test, and chisquare tests were performed to identify the risk factors of SCF. Variables with a p-value $<0.25$ in the univariate analysis were included in the multivariate logistic regression analysis. Odds Ratios (ORs) and 95\% Confidence Intervals (CIs) were taken as risk measurements in the multivariate logistic regression model. Receiver Operating Characteristic (ROC) analysis was used to determine the sensitivity and specificity of each biomarker and to determine the cut-off values to discriminate subjects with SCF. ROC curves are given for biomarkers with a statistically significant difference between the SCF and NCF (control) groups. A p-value $<0.05$ was considered to indicate statistical significance in all analyses.

\section{Results}

The SCF and NCF groups included 54 and 35 subjects, respectively. The baseline characteristics of all study samples are shown in Table 1.

There were more smokers in the SCF group $(p<0.013)$. The levels of serum endocan and high-sensitivity $\mathrm{C}$-reactive protein (hsCRP) were also higher in SCF patients relative to controls $(p=0.009 ; p<0.001$, respectively) (Figure 1$)$. The serum lymphocyte count was lower in the SCF group $(p=0.005)$. The TFC of each coronary artery and the mean TFC were higher in SCF subjects $(p<0.001)$. The other baseline characteristics of 
the study population did not differ significantly between groups (Table 1).

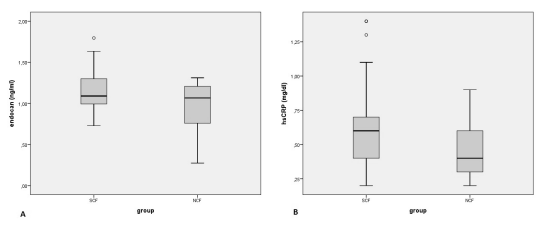

Figure 1. Boxplot graphics for endocan (A) and high-sensitivity $C$ reactive protein (hsCRP) (B) biomarkers

In total, $11(20.4 \%)$ subjects had SCF in three arteries, 17 $(31.4 \%)$ had SCF in two arteries, and $26(48.2 \%)$ had SCF in only one artery. The frequency of SCF according to coronary vessel was as follows: 39 subjects $(72 \%)$ in the LAD, 26 subjects (48\%) in the RCA, and 21 subjects (48\%) in the LCX. The serum endocan concentration was higher in patients with SCF in three vessels than in those with SCF in one or two vessels $(12.8 \pm 2.2$ and $11.0 \pm 2.0$, respectively; $\mathrm{p}=0.016)$.

According to the correlation analysis, hsCRP and endocan levels showed a significant positive correlation with mean TIMI frame count variables $(\mathrm{p}<0.05)$. Scatter plots were used to reveal the association between pairs of variables. Table 2 shows the results from the correlation analysis and Figure 2 shows the scatter plots of these correlations.
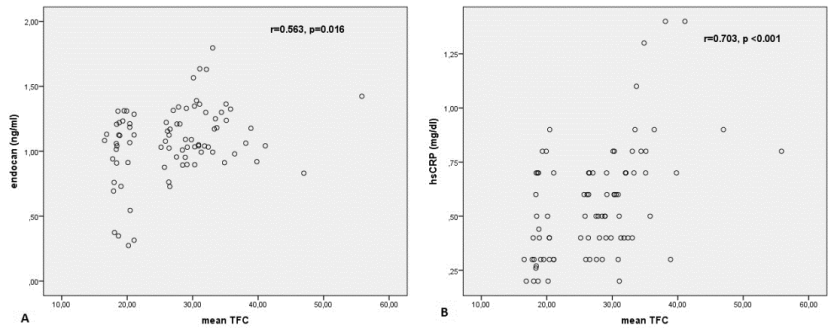

Figure 2. Scatter plots of correlations of endocan $(A)$ and hsCRP (B) biomarkers with mean variables.

Multivariate logistic regression analysis was used to identify the major risk factors of SCF. The Neutrophil-to-Lymphocyte Ratio (NLR), platelet and lymphocyte counts, body mass index, and smoking status were examined in the logistic regression analysis, along with the endothel biomarkers endocan, hrCRP, and sTM. All risk factors and endothel biomarkers were included in the univariate logistic regression analysis. In the multivariate logistic regression analysis, the backward Wald method was used for variable selection. Two of the endothel biomarkers (hsCRP and endocan) were analysed along with smoking status, NLR, and platelet count. A higher NLR and smoking were identified as the major risk factors of SCF (OR for NLR: 15.435, 95\% CI: 2.608-91.331, p<0.05; OR for smoke: $4.523,95 \% \mathrm{CI}$ : 1.107-18.481, $\mathrm{p}<0.05)$. hsCRP and endocan were also identified as risk factors of SCF (OR for hsCRP: 1.045, 95\% CI: 1.011-1.079, $\mathrm{p}<0.05$; OR for endocan: $1.387,95 \%$ CI: $1.039-1.85, \mathrm{p}<0.05)$. Table 3 shows the results of the logistic regression analysis.

ROC analysis was performed to determine the discriminatory capacity of endocan and hsCRP levels. The area under the curve (AUC) value for hsCRP was $0.714 \quad(95 \%$ CI: $0.592-0.835 ; \mathrm{p}<0.001)$. With the cut-off value of 0.47 , the sensitivity and specificity values were $70.4 \%$ and $65.5 \%$, respectively. The AUC value for endocan was 0.622 (95\% CI: $0.495-0.749 ; \mathrm{p}<0.068)$. With the cut-off value of 7.61 , the sensitivity and specificity values were $98.1 \%$ and $27.6 \%$, respectively. Figure 3 shows the ROC curves for these two biomarkers.
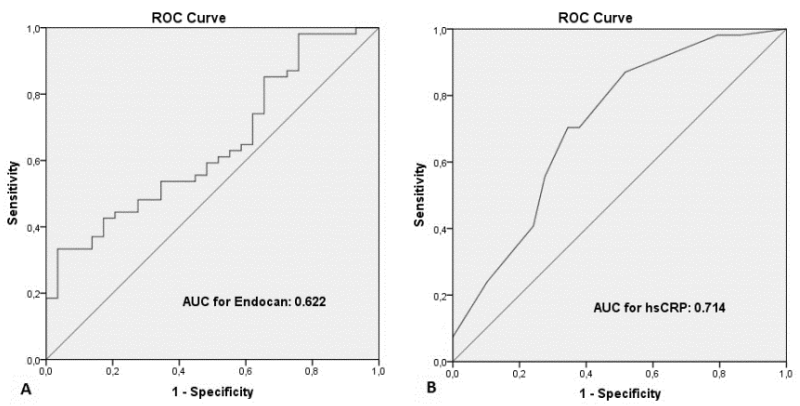

Figure 3. Receiver operating characteristic (ROC) curve analysis for endocan (A) and hsCRP (B) biomarkers.

Table 1. Baseline clinical, laboratory and angiographic characteristics of the study population.

\begin{tabular}{|c|c|c|c|}
\hline & $\operatorname{SCF}(n=54)$ & NCF $(n=35)$ & p \\
\hline \multicolumn{4}{|l|}{ Clinical data } \\
\hline Age, y & $53 \pm 9$ & $53 \pm 4$ & 0.993 \\
\hline Female, n (\%) & $16(29)$ & $12(34)$ & 0.334 \\
\hline BMI, $\mathrm{kg} / \mathrm{m}^{2}$ & $28.7 \pm 3.2$ & $27.8 \pm 1.7$ & 0.181 \\
\hline Diabetes mellitus, n (\%) & $18(33)$ & $9(25)$ & 0.831 \\
\hline Hypertension, n (\%) & $25(46)$ & $14(40)$ & 0.863 \\
\hline Smoking, n (\%) & $28(51)$ & $7(20)$ & 0.013 \\
\hline \multicolumn{4}{|l|}{ Laboratory data } \\
\hline Total cholesterol, mg/dl & $200.9 \pm 47.0$ & $196.5 \pm 24.4$ & 0.642 \\
\hline LDL, mg/dl & $110.2 \pm 32.7$ & $100.1 \pm 17.8$ & 0.124 \\
\hline $\mathrm{HDL}, \mathrm{mg} / \mathrm{dl}$ & $39.5 \pm 8.7$ & $39.2 \pm 5.7$ & 0.902 \\
\hline Triglyceride, mg/dl & $234(139-398)$ & $203(117-312)$ & 0.654 \\
\hline Fasting glucose, mg/dl & $107.5 \pm 22.4$ & $101.4 \pm 28.7$ & 0.287 \\
\hline Creatinine, mg/dl & $0.82 \pm 0.11$ & $0.78 \pm 0.08$ & 0.313 \\
\hline Hemoglobin, g/dl & $15.0 \pm 1.5$ & $15.2 \pm 1.0$ & 0.613 \\
\hline WBC, $10^{3} / \mathrm{mm}^{3}$ & $8.58 \pm 1.53$ & $8.93 \pm 1.35$ & 0.313 \\
\hline Neutrophil, $10^{3} / \mathrm{mm}^{3}$ & $4.98 \pm 1.13$ & $4.76 \pm 0.82$ & 0.355 \\
\hline Lymphocyte, $10^{3} / \mathrm{mm}^{3}$ & $2.73 \pm 0.66$ & $3.20 \pm 0.77$ & 0.005 \\
\hline NLR, \% & $1.88 \pm 0.52$ & $1.55 \pm 0.35$ & 0.003 \\
\hline Platelet, $10^{3} / \mathrm{mm}^{3}$ & $239.6 \pm 52.3$ & $262.4 \pm 42.4$ & 0.098 \\
\hline Endocan, ng/ml & $1.14 \pm 0.22$ & $0.96 \pm 0.32$ & 0.009 \\
\hline sTM, pg/ml & $657.06 \pm 198.18$ & $592.76 \pm 128.45$ & 0.119 \\
\hline
\end{tabular}




\begin{tabular}{llll}
\hline hsCRP, mg/dl & $0.60(0.2-1.4)$ & $0.40(0.2-0.9)$ & $<0.001$ \\
\hline $\begin{array}{l}\text { TIMI frame } \\
\text { measurements }\end{array}$ & count & & \\
\hline LAD (corrected) & $34.6 \pm 6.8$ & $21.0 \pm 1.0$ & $<0.001$ \\
\hline CX & $31.9 \pm 9.4$ & $18.1 \pm 2.3$ & $<0.001$ \\
\hline RCA & $30.0 \pm 9.8$ & $18.1 \pm 1.8$ & $<0.001$ \\
\hline Mean & $31.5 \pm 5.5$ & $19.0 \pm 1.2$ & $<0.001$ \\
\hline
\end{tabular}

BMI: Body Mass Index, HDL: High-Density Lipoprotein, hsCRP: High Sensitive C-Reactive Protein, LDL: Low-Density Lipoprotein, NLR: Neutrophil-to-Lymphocyte Ratio, sTM: soluble Thrombomodulin, WBC White Blood Cell.

Table 2. Correlation coefficients between endocan and the mean TIMI frame count variable.

\begin{tabular}{lll}
\hline Variables & Correlation coefficients $(\mathbf{r})^{* *}$ & $\mathbf{p}$ \\
\hline hsCRP & 0.703 & $<0.001$ \\
\hline sTM & 0.092 & 0.41 \\
\hline Endocan & 0.563 & 0.016 \\
\hline
\end{tabular}

** Spearman Correlation Coefficient was used for all biomarkers. hsCRP: High Sensitive C-Reactive Protein, sTM: soluble Thrombomodulin.

Table 3. Univariate and multivariate logistic regression analyses.

\begin{tabular}{lllll}
\hline & Univariate & & Multivariate & \\
\cline { 2 - 5 } & OR (95\% Cl) & $\mathbf{p}$ & OR (95\% CI) & p \\
\hline hsCRP & $1.015(1.007-1.024)$ & 0.001 & $1.045(1.011-1.079)$ & 0.008 \\
\hline Endocan & $1.072(1.028-1.118)$ & 0.007 & $1.387(1.039-1.85)$ & 0.026 \\
\hline NLR & $1.546(1.193-2.005)$ & 0.001 & $15.435(2.608-91.331)$ & 0.003 \\
\hline Smoking & $3.385(1.24-9.239)$ & 0.017 & $4.523(1.107-18.481)$ & 0.036 \\
\hline Platelet & $1.021(1.003-1.039)$ & 0.022 & $0.862(0.763-0.974)$ & 0.017 \\
\hline Lymphocyte & $1.015(1.000-1.030)$ & 0.046 & & \\
\hline sTM & $1.009(1.002-1.016)$ & 0.017 & & \\
\hline BMI & $1.029(1.007-1.039)$ & 0.005 & & \\
\hline
\end{tabular}

BMI: Body Mass Index; hsCRP: high sensitive C-Reactive Protein; NLR Neutrophil-to-Lymphocyte Ratio; sTM: soluble Thrombomodulin.

\section{Discussion}

In this report, serum endocan levels were significantly higher in SCF patients than in controls; higher STM levels were also observed, although the difference did not reach statistical significance. We also demonstrated that serum endocan level was correlated with the degree of SCF. Only two previous studies have investigated the relationship between serum endocan level and SCF; to best of our knowledge, this was the first study to focus on the level of sTM as a novel marker of endothelial dysfunction in SCF patients $[10,11]$.

Although several studies have suggested the prognostic value of SCF for major adverse cardiovascular events, the pathophysiology of SCF remains unclear. Several mechanisms, such as endothelial dysfunction, diffuse atherosclerosis, and coronary vasomotor dysfunction, have been associated with this clinical entity $[3,4,12]$.

There is growing evidence for a role of endothelial dysfunction in the etiology of SCF. Sezgin et al. [3] showed reduced endothelium-dependent Flow-Mediated Dilatation (FMD) of the brachial artery in SCF patients, supporting the endothelial dysfunction hypothesis in the etiology of SCF. Previous studies on endothelial dysfunction markers in SCF reported that increased levels of plasma endothelin-1, homocysteine, and asymmetric dimethylarginine, and decreased levels of plasma adiponectin and nitric oxide, were responsible for the etiopathogenesis of SCF [13-16].

Endocan is an endothelium-derived soluble proteoglycan that is believed to play a role in the pathogenesis of endothelial diseases, such as hypertension, atherosclerosis, and obstructive sleep apnea $[6,17,18]$. Moreover, significantly higher endocan levels were detected in subjects with vascular-Behçet disease [19]. In our study, the endocan concentration was higher in SCF patients than in controls, confirming previous reports $[10,11]$.

According to our multivariate logistic regression analysis, circulating endocan was identified as an independent predictor of SCF. A powerful correlation between TFC value and endocan level, and a higher endocan concentration in subjects with SCF involving three vessels versus two vessels or one vessel, allowed us to conclude that endocan may serve as a promising biomarker for the detection and evaluation of SCF. We hypothesize that endocan activates adhesion molecules and increases the secretion of potent chemokines, leading to endothelial dysfunction. It may also enhance the stimulation of vascular smooth muscle proliferation and migration, which could lead to atherosclerosis via neointima formation and, ultimately, SCF [20]. Further studies will help elucidate the exact mechanism.

TM is an integral protein that is widely detected on the endothelial lumen of all vascular structures and is believed to play a role in coagulation, inflammation, and endothelial dysfunction [21]. During endothelial pathologies, TM is released from the endothelium and can be detected in the serum in its soluble form. A significant increase in STM level has been reported in vascular diseases, such as atherosclerosis, cardioembolic stroke, and sepsis-associated disseminated intravascular coagulation, indicating that STM may represent a useful marker to assess endothelial dysfunction and vascular risk [22,23]. We also found increased levels of sTM in SCF subjects relative to controls, although the difference did not reach statistical significance.

Several studies have suggested that inflammation may lead to SCF. Li et al. [24] reported increased levels of hsCRP and interleukin-6 in SCF patients. Another inflammatory parameter, NLR, was also reported to be correlated with SCF [25]. Supporting previous reports, higher concentrations of hsCRP and NLR were found in the SCF group relative to controls in our study. These factors were also identified as 
independent predictors of SCF according to our multivariate analysis.

\section{Limitations}

The relatively small sample size was the main limitation of our study. Although the sample size was sufficient to detect a significant association between endocan and SCF, we were not able to prove an association between SCF and STM. The crosssectional design was another limitation of the study. Lastly, we did not use intravascular ultrasound or optical coherence tomography to detect cases with normal coronary arteries. Rather, our diagnosis depended on the visible angiograms, which may have biased the randomization of our study population.

\section{Conclusion}

We demonstrated that the endocan concentration was significantly higher in SCF patients than in controls and positively correlated with the severity of SCF. Moreover, sTM levels were increased in SCF patients, although the difference did not reach statistical significance. These findings suggest that the endocan level may serve as an indicator to determine the presence and extent of SCF. Further larger investigations are needed to determine the exact role of both endocan and sTM in the pathophysiology of SCF.

\section{Acknowledgments}

We thank the staff of Cathater Laboratories, Şanlıurfa Mehmet Akif İnan Training and Research Hospital, and Harran University (Faculty of Medicine and Department of Cardiology).

\section{References}

1. Mangieri E, Macchiarelli G, Ciavolella M, Barillà F, Avella A, Martinotti A, DellItalia LJ, Scibilia G, Motta P, Campa PP. Slow coronary flow: clinical and histopathological features in patients with otherwise normal epicardial coronary arteries. Cathet Cardiovasc Diagn 1996; 37: 375-371.

2. Hawkins BM, Stavrakis S, Rousan TA, Abu-Fadel M, Schechter E. Coronary slow flow-prevalence and clinical correlations. Circ J 2012; 76: 936-942.

3. Sezgin AT, Sigirci A, Barutcu I, Topal E, Sezgin N, Ozdemir R, Yetkin E, Tandogan I, Kosar F, Ermis N, Yologlu S, Bariskaner E, Cehreli S. Vascular endothelial function in patients with slow coronary flow. Coron Artery Dis 2003; 14: 155-161.

4. Aksan G, Soylu K, Aksoy O, Özdemir M, Yanik A, Yuksel S, Gedikli O, Gulel O, Sahin M. The relationship between neutrophil gelatinase-associated lipocalin levels and the slow coronaryflow phenomenon. Coron Artery Dis 2014; 25: 505-509.

5. Sarrazin S, Adam E, Lyon M, Depontieu F, Motte V, Landolfi C, Lortat-Jacob H, Bechard D, Lassalle P,
Delehedde M. Endocan or endothelial cell specific molecule-1 (ESM-1): a potential novel endothelial cell marker and a new target for cancer therapy. Biochim Biophys Acta 2006; 1765: 25-37.

6. Aparci M, Isilak Z, Uz O, Yalcin M, Kucuk U. Endocan and endothelial dysfunction. Angiology 2015; 66: 488-489.

7. Sadler JE. Thrombomodulin structure and function. Thromb Haemost 1997; 78: 392-395.

8. Strijbos MH, Rao C, Schmitz PI, Kraan J, Lamers CH, Sleijfer S, Terstappen LW, Gratama JW. Correlation between circulating endothelial cell counts and plasma thrombomodulin levels as markers for endothelial damage. Thromb Haemost 2008; 100: 642-647.

9. Gibson CM, Cannon CP, Daley WL, Dodge JT Jr, Alexander B Jr, Marble SJ, McCabe CH, Raymond L, Fortin T, Poole WK, Braunwald E. TIMI frame count: a quantitative method of assessing coronary artery flow. Circulation 1996; 93: 879-888.

10. Kundi H, Gok M, Kiziltunc E, Topcuoglu C, Cetin M, Cicekcioglu H, Ugurlu B, Ulusoy FV. The relationship between serum endocan levels with the presence of slow coronary flow: a cross-sectional study. Clin Appl Thromb Hemost 2015.

11. Ye MF, Zhao ZW, Luo YK, Dong XF, Yan YM. Elevated endocan concentration is associated with coronary slow flow. Scand J Clin Lab Invest 2016; 16: 1-4.

12. Wang X, Nie SP. The coronary slow flow phenomenon: characteristics, mechanisms and implications. Cardiovasc Diagn Ther 2011; 1: 37-43.

13. Camsari A, Pekdemir H, Cicek D, Polat G, Akkus MN, Doven O, Cin VG, Katircibasi T, Parmaksiz T. Endothelin-1 and nitric oxide concentrations and their response to exercise in patients with slowcoronary flow. Circ J 2003; 67: 1022-1028.

14. Barutcu I, Sezgin AT, Sezgin N, Gullu H, Esen AM, Topal E, Ozdemir R. Elevated plasma homocysteine level in slow coronary flow. Int J Cardiol 2005; 101: 143-145.

15. Selcuk MT, Selcuk H, Temizhan A, Maden O, Ulupinar H, Baysal E, Ozeke O, Sasmaz A. Asymmetric dimethylarginine plasma concentrations and L-arginine/ asymmetric dimethylarginine ratio in patients with slow coronary flow. Coron Artery Dis 2007; 18: 545-551.

16. Selcuk H, Selcuk MT, Temizhan A, Maden O, Saydam GS, Ulupinar H, Dogan M, Aydin C, Topcu DI, Sasmaz A. Decreased plasma concentrations of adiponectin in patients with slow coronary flow. Heart Vessels 2009; 24: 1-7.

17. Wang XS, Yang W, Luo T, Wang JM, Jing YY. Serum endocan levels are correlated with the presence and severity of coronary artery disease in patients with hypertension. Genet Test Mol Biomarkers 2015; 19: 124-127.

18. Altintas N, Mutlu LC, Akkoyun DC, Aydin M, Bilir B, Yilmaz A, Malhotra A. Effect of CPAP on new endothelial dysfunction marker, endocan, in people with obstructive sleep apnea. Angiology. 2016; 67: 364-374.

19. Balta I, Balta S, Koryurek OM, Demirkol S, Mikhailidis DP, Celik T, Cakar M, Kucuk U, Eksioglu M, Kurt YG. 
Serum endocan levels as a marker of disease activity in patients with Behçet disease. J Am Acad Dermatol 2014; 70: 291-296.

20. Lee W, Ku SK, Kim SW, Bae JS. Endocan elicits severe vascular inflammatory responses in vitro and in vivo. J Cell Physiol 2014; 229: 620-630.

21. Martin FA, Murphy RP, Cummins PM. Thrombomodulin and the vascular endothelium: insights into functional, regulatory, and therapeutic aspects. Am J Physiol Heart Circ Physiol 2013; 304: 1585-1597.

22. Dharmasaroja P, Dharmasaroja PA, Sobhon P. Increased plasma soluble thrombomodulin levels in cardioembolic stroke. Clin Appl Thromb Hemost 2012; 18: 289-293.

23. Lin SM, Wang YM, Lin HC, Lee KY, Huang CD, Liu CY, Wang CH, Kuo HP. Serum thrombomodulin level relates to the clinical course of disseminated intravascular coagulation, multiorgan dysfunction syndrome, and mortality in patients with sepsis. Crit Care Med 2008; 36: 683-689.
24. Li JJ, Qin XW, Li ZC, Zeng HS, Gao Z, Xu B, Zhang CY, Li J. Increased plasma C-reactive protein and interleukin-6 concentrations in patients with slowcoronary flow. Clin Chim Acta 2007; 385: 43-47.

25. Dogan M, Akyel A, Cimen T, Bilgin M, Sunman H, Kasapkara HA, Arslantas U, Yayla KG, Acikel S, Yeter E. Relationship between neutrophil to lymphocyte ratio and slow coronary flow. Clin Appl Thromb Hemostasis 2015; 21: $251-254$

\section{*Correspondence to}

Sadettin Selçuk Baysal

Cardiology Department

Şanlıurfa

Turkey 